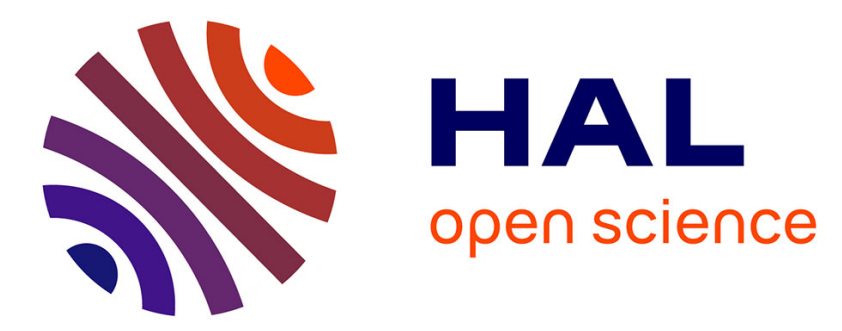

\title{
Dimensioning of the downlink in OFDMA cellular networks via an Erlang's loss model
}

Bartlomiej Blaszczyszyn, Mohamed Kadhem Karray

\section{To cite this version:}

Bartlomiej Blaszczyszyn, Mohamed Kadhem Karray. Dimensioning of the downlink in OFDMA cellular networks via an Erlang's loss model. European Wireless, May 2009, Aalborg, Denmark. 10.1109/EW.2009.5358006 . inria-00439663

\section{HAL Id: inria-00439663 https://hal.inria.fr/inria-00439663}

Submitted on 8 Dec 2009

HAL is a multi-disciplinary open access archive for the deposit and dissemination of scientific research documents, whether they are published or not. The documents may come from teaching and research institutions in France or abroad, or from public or private research centers.
L'archive ouverte pluridisciplinaire HAL, est destinée au dépôt et à la diffusion de documents scientifiques de niveau recherche, publiés ou non, émanant des établissements d'enseignement et de recherche français ou étrangers, des laboratoires publics ou privés. 


\title{
Dimensioning of the downlink in OFDMA cellular networks via an Erlang's loss model
}

\author{
Bartłomiej Błaszczyszyn $^{1}$ and Mohamed Kadhem Karray ${ }^{2}$
}

\begin{abstract}
In this paper we propose the following approach to the dimensioning of the radio part of the downlink in OFDMA networks. First, we use information theory to characterize the bitrate in the channel from a base station to its mobile. It depends on the power and bandwidth allocated to this mobile. Then, we describe the resource (power and bandwidth) allocation problem and characterise feasible configurations of bit-rates of all users. As the key element, we propose some particular sufficient condition (in a multi-Erlang form) for a given configuration of bit-rates to be feasible. Finally, we consider an Erlang's loss model, in which streaming arrivals whose admission would lead to the violation of this sufficient condition are blocked and lost. In this model, the blocking probabilities can be calculated using Kaufman-Roberts algorithm. We propose it to evaluate the minimal density of base stations assuring acceptable blocking probabilities for a streaming traffic of a given load per surface unit. We validate this approach by comparison of the blocking probabilities to these simulated in the similar model in which the admission control is based on the original feasibility property (instead of its sufficient condition). Our sufficient bit-rate feasibility condition can also be used to dimension the network with respect to the elastic traffic.
\end{abstract}

\section{INTRODUCTION}

Orthogonal Frequency-Division Multiple Access (OFDMA) is a multi-user version of the orthogonal frequency-division multiplexing (OFDM) digital modulation scheme. Multiple access is achieved by assigning subsets of sub-carriers to individual users. This allows simultaneous transmission to and from several users. The recent interest in OFDMA comes from the fact that it is used in the mobility mode of IEEE 802.16 WirelessMAN Air Interface standard, commonly referred to as WiMAX and OFDMA is currently a working assumption in 3GPP Long Term Evolution (LTE) downlink. Also, OFDMA is the candidate access method for the IEEE 802.22 Wireless Regional Area Networks. It is the context of LTE cellular networks that we have primarily on mind in this paper, however, our approach applies to other OFDMA downlink scenarios as well.

Our objective is to build a dimensioning method for the radio part of the downlink in wireless cellular OFDMA networks, i.e.; a method allowing to evaluate what is the minimal density of base stations assuring a given quality of service (QoS) of a given traffic demand per surface unit. This requires construction of some model that describes the relation between the three key parameters: traffic demand per surface unit, cell size and the $Q o S$. In general, such a model is not easy to establish for a cellular network since it needs to account for the geographic positions of the users and the evolution of these positions due to the dynamics of calls arrivals and departures. Moreover, in the case of OFDMA, it needs to account for the power and bandwidth allocation mechanisms specific for this technology. At the same time, it needs to represent some reasonable simplification of the reality, since any too "realistic" model has to be evaluated

\footnotetext{
${ }^{1}$ INRIA \& Ecole Normale Supérieure and Math. Inst., Univ. of Wrocław, 45 rue d'Ulm, 75005 Paris France, Bartek.Blaszczyszyn @ens.fr,

${ }^{2}$ France Télécom Research and Development, 38/40 rue du Général Leclerc, 92794 Issy-Moulineaux France, mohamed.karray@orange-ftgroup.com
}

by pure simulation. This however is usually not appropriate for dimensioning purpose since evaluating the network under one particular choice of parameters requires long simulations.

OFDMA based network (like LTE and WiMAX) can serve streaming and elastic traffic. Streaming (real-time) traffic consists of connections of some given duration that require some given bit-rate. Elastic traffic (non-real-time) consists of connections aiming to transfer some given volume of data at a rate that may be decided by the network and lasting until the completion of this job. The QoS of the streaming traffic is typically evaluated in terms of the call blocking probabilities, while the QoS of the elastic traffic can be evaluated in the (mean) transfer time of a unit volume or the mean transmission speed, called throughput.

Queueing theory provides some "classical" models (as Erlang's loss systems, processor sharing queues, etc.) that allow to evaluate these QoS parameters. They have been successfully used to dimension circuit-switched and other communication networks. In general, it is not however obvious how to use these models to dimension the radio part of wireless cellular networks.

In this paper we show how the downlink in LTE OFDMA cellular network can be dimensioned by means of a mutlirate Erlang's loss model that takes into account informationtheoretic foundations of the wireless communications.

The remaining part of this paper is organized as follows. In the next section we outline our approach to the problem and discuss briefly the related work. Our model of the OFDMA network is presented in Section III. In Section IV we study the problem of feasible bit-rate assignments in this model. The results are used to construct an Erlang's loss model in Section V, in which we also numerically validate the whole approach.

\section{OUTLINE OF THE PROPOSED APPROACH}

We want to find an intrinsic relation between the traffic demand, network cell size and users QoS. For this reason, instead of studying an arbitrary OFDMA network control scheme, we look at some "reference" one based on a theoretic feasibility of the resource allocation. Roughly speaking, it admits a new steaming user if and only if according to the information theoretic limitations on the feasible bit-rates, it can be served by the available resources of the network with already present users (possibly, at the price of some decrease of the bit-rates of the elastic users).

This control scheme, whose performance has to be studied by simulation, will be considered as the reference for constructing other schemes, including some particular one that can be evaluated analytically and thus can be used for the purpose of the network dimensioning. The QoS induced by these conditions will be shown to be close to the reference one.

\section{A. Resource allocation and feasible bit-rates in OFDMA}

The resource to be shared and allocated in OFDMA network consists of the power and the bandwidth. More specifically, on the downlink, each base station (BS) should allocate a power and 
a bandwidth (sub-carriers) to each user in its cell. In doing so, the BS should respect the constraints on the maximal total power and total bandwidth called power and bandwidth constraints respectively. Once the power and bandwidth allocation is given, the bit-rate $r$ of each user may not exceed some function $f$ of the signal-to-noise-and-interference ratio (SINR), that is $r \leq f(\mathrm{SINR})$. The function $f$ depends on the assumptions on the channel between BS and its users. For example in case of the additive white gaussian noise (AWGN) channel with single user detection this function is given by the celebrated Shannon relation $f(\mathrm{SINR})=w \log _{2}(1+\mathrm{SINR})$ where $w$ is the bandwidth. This constraint on $r$ is called the information theory constraint.

We say that a vector of user bit-rates is feasible if it satisfies the information theory constraint for some resource (power and bandwidth) allocation satisfying their respective constraints. Verification of the feasibility of bit-rates is the basis of our "reference" network control. We will also propose some sufficient condition for the feasibility problem, whose form allows for analytical evaluation of the QoS in the network when it is used for admission and congestion control.

\section{B. Multi-rate Erlang loss model and its blocking probabilities}

Users arrive to the network and request some streaming or elastic service. A (bit simplified) vision of the network control that we have in this paper is that only streaming users are blocked. Specifically, they are blocked when their acceptance would lead to a configuration not satisfying some given admission condition. The role of this condition is to guarantee the required service for all already present (streaming) users. Elastic users are always accepted and their bit-rates are assigned by the network (by optimizing some fair objective function) after satisfying the streaming users. The QoS perceived by the users may be quantified under some particular probabilistic assumptions on the traffic demand by the blocking probability for streaming calls and mean throughput of elastic calls.

Assuming some Markov traffic model (so called free model) and considering an admission condition being some truncation of the state space of this model, under very general conditions one can express the blocking probabilities in terms of the stationary distribution of the free model. Namely, as the probability, that the free process is close to the boundary of the truncated part of the space; ${ }^{3}$. However, for a complicated admission condition (complicated form of the space truncation) it is difficult or even impossible to evaluate analytically this probability, even in the (most simple) Poisson case of the stationary distribution of the free process. This difficulty appears when the feasibility of bitrates is taken as the admission condition (i.e., in the reference control scheme).

To cope with this problem, one has to look for some special forms of the admission condition, where, under Poisson stationary distribution of the free process, the evaluation of the blocking probability simplifies considerably. This is the case, when the admission condition has a multi-Erlang form; i.e., when it can be written as the weighted sum of the bit-rates of users not exceeding some constant. This particular form permits to calculate the blocking probabilities using the Kaufman-Roberts

\footnotetext{
${ }^{3}$ A classical example for this more general relation is the famous Erlang's loss formula giving the blocking probability for $M / G / c / c$ loss queue with $c$ servers and the total capacity of $c$ users as the probability that Poisson number of users in the stationary $M / G / \infty$ (free) queue is equal to $c$.
}

algorithm [13, 20]. Moreover, assuming some appropriate separation of the time scale of the coexisting streaming and elastic traffic [7], one can also evaluate the mean throughput of the elastic traffic using a multi-class processor sharing model (see for example [5]).

The goal of this paper is to propose some reasonable sufficient condition for the feasibility of the bit-rates in the multi-Erlang form, use it as the admission condition in some Erlang's loss model and evaluate the corresponding blocking probabilities. By reasonable we mean inducing a loss of network capacity up to about $10 \%$ for blocking probability close to 0.02 , with respect to the reference network control scheme based on the exact feasibility admission condition. ${ }^{4}$

\section{Related work}

For a general reference on OFDMA see [17] and the references therein. A growing interest in OFDMA has resulted in many publications proposing various efficient methods for resource allocation in OFDMA. Here are some recent and/or highly referenced ones [1, 9, 14, 15, 19, 21, 22]; ${ }^{5}$. In general it is however extremely difficult to evaluate the QoS offered by the network with these methods implemented. Many such studies consider the case of a single cell, e.g. [11], [23]. The muti-cell case is studied in [10] and [18]. In [8] different frequency reuse schemes are compared.

The present work adopts the approach proposed in [6] (with a background in $[2,3,12]$ ) that is implemented in the dimensioning tool of Orange. It consists in proposing some "virtual" network control mechanism (not necessarily meant to be implemented in network controllers) that however is simple enough and can by studied by the classical tools of queueing theory. The QoS imposed by this condition is shown to be close to this of some reference network control, to which "real" control schemes should be close too.

\section{MUlTi-CELl OFDMA DOWNLINK MODEL}

\section{A. Model assumptions}

We will consider a wireless network composed of several base stations (BS). Each BS is equipped with a single antenna (no MIMO) and its total power is limited to some given maximal value. The same frequency spectrum is available to all BS (frequency reuse factor equal to one). Each BS allocates disjoint sub-carriers to its users without macrodiversity (each user is served by exactly one BS). Thus, any given user receives only other-BS interference that is the sum of powers emitted by other $\mathrm{BS}$ on the sub-carriers allocated to him by his BS. The number of interfering BS is large and it is reasonable to assume that the interference power spectral density is constant in the whole spectrum. ${ }^{6}$ Users perform single user detection; the interference is considered as noise. Efficient (e.g. turbo) codes are used to obtain bit-rates close to the Shannon limit. In our analysis we neglect fading effects. However, in the case of non-opportunistic scheduling, the Rayleigh fading may be tentatively taken into account in our approach by an appropriate modification of the AWGN capacity formula that consists in dividing the SINR by 2

\footnotetext{
${ }^{4}$ This loss of capacity seems to be acceptable for network operators looking for rapid network dimensioning tools. Note also that it is evaluated with respect to the reference network performance assuming some perfect control scheme.

${ }^{5}$ Google Scholar presents about 2500 recent publications on the subject "resource+allocation+OFDMA".

${ }^{6} \mathrm{~A}$ suitable fast sub-carrier permutation (for a given configuration of users) may give a further justification of this assumption.
} 
(cf [16]). In the case of opportunistic scheduling, further studies are necessary to account for the fading effect.

\section{B. Model description}

The network is composed of a finite set $U$ of BS located on the plane. We assume that each BS serves users is some exclusive geometric cell associated to it, which does not evolve in time. At present, the geometry of the network is not important; it will be specified in Section V-C1. Lets denote by $L_{u, m}$ the propagationloss between a BS $u$ and a given user $m$. We will write $m \in u$ when user $m$ is served by base station $u$.

Each BS $u$ transmits a power $P_{u, m}$ to each $m \in u$. The total transmitted power is

$$
P_{u}=P_{u}^{\prime}+\sum_{m \in u} P_{u, m}
$$

where $P_{u}^{\prime}$ designates the power of common channels (not dedicated to a specific user). The maximal value of the total transmitted power is denoted $\tilde{P}_{u}$. We assume that

$$
P_{u}^{\prime}=\epsilon \tilde{P}_{u}
$$

for some given constant $0<\epsilon<1$.

Each BS $u$ allocates some number of sub-carriers of the total width $w_{m}$ from the total spectrum of width $W$ to each user $m \in u$, in such a way that two different users of the same BS have disjoint subsets of sub-carriers. However, since the same frequency spectrum is allocated (assumed on average uniformly) by all $\mathrm{BS}$, user $m \in u$ receives interference from each base station $v \neq u$ of power $\frac{w_{m}}{W} P_{v} / L_{v, m}$. We assume that this interference acts as Gaussian noise, thus we assume that the SINR of user $m \in u$ is equal to

$$
\operatorname{SINR}_{m}=\frac{P_{u, m} / L_{u, m}}{w_{m} N_{0}+\frac{w_{m}}{W} \sum_{v \neq u} P_{v} / L_{v, m}}
$$

where $N_{0}$ is the power spectral density of the thermal noise. The bit-rate of user $m$ is denoted by $r_{m}$.

\section{Resource allocation constraints}

All powers, bandwidths and bit-rates should be nonnegative, but we will not write this explicitely. The power constraint is

$$
P_{u} \leq \tilde{P}_{u}, \quad u \in U
$$

The bandwidth constraint is

$$
\sum_{m \in u} w_{m} \leq W, \quad u \in U
$$

As a consequence of our channel assumption the information theory constraint is

$$
r_{m} \leq w_{m} \log _{2}\left(1+\operatorname{SINR}_{m}\right), \quad m \in u, u \in U
$$

Definition 3.1: We will say that a vector of user bit-rates $\left(r_{m}\right)$ is feasible if there exist powers $\left(P_{u, m}\right)$ and bandwidths $\left(w_{m}\right)$ such that the constraints (3), (4) and (5) are satisfied. In this case we will also say that $\left(r_{m}\right)$ satisfies the feasibility condition (FC).

The problem of feasibility of a given vector of bit-rates is crucial for our approach to network dimensioning. In the following section we will give some more explicit sufficient conditions allowing to say that some bit-rates are feasible.

\section{BIT-RATE FEASIBILITY CONDITIONS}

In order to propose some analytical approach to network dimensioning, in what follows we propose some more explicit sufficient conditions for the feasibility of the bit-rates.

\section{A. When all BS emit with their maximal powers}

In this section we will assume that all the BS use their maximal powers; i.e., the condition (3) is replaced by a more constraining one

$$
P_{u}=\tilde{P}_{u} \quad u \in U
$$

In view of (1), this is only possible when each base station has at least one user, so we assume it implicitly when assumption (6) is made.

Definition 4.1: We will say that a given vector of user bitrates $\left(r_{m}\right)$ is feasible at maximal power if the there exist powers $\left(P_{u, m}\right)$ and bandwidths $\left(w_{m}\right)$ satisfying (6), (4) and (5).

Denote for $m \in u, u \in U$

$$
\begin{aligned}
f(m) & =\sum_{v \neq u} \frac{L_{u, m}}{L_{v, m}} \frac{\tilde{P}_{v}}{\tilde{P}_{u}} \\
f^{\prime}(m) & =\frac{1}{1-\epsilon}\left(\frac{W N_{0} L_{u, m}}{\tilde{P}_{u}}+f(m)\right), \quad m \in u, u \in U \\
\xi_{m} & =\frac{w_{m}}{W}\left(2^{r_{m} / w_{m}}-1\right)
\end{aligned}
$$

Proposition 4.2: A vector of user bit-rates $\left(r_{m}\right)$ is feasible at maximal power iff there exist bandwidths $\left(w_{m}\right)$ satisfying (4) and the following condition

$$
\sum_{m \in u} f^{\prime}(m) \xi_{m} \leq 1, \quad u \in U
$$

Moreover, for any bandwidth allocation $\left(w_{m}\right)$ which satisfies (4) and (10) the following power allocation satisfies (6) and (5)

$$
P_{u, m}=\frac{f^{\prime}(m) \xi_{m}}{\sum_{n \in u} f^{\prime}(n) \xi_{n}}(1-\epsilon) \tilde{P}_{u}
$$

Proof: Under condition (6), the information theory constraint (5) may be rewritten equivalently as

$$
P_{u, m} \geq f^{\prime}(m) \xi_{m}(1-\epsilon) \tilde{P}_{u} \quad m \in u, u \in U
$$

where $f^{\prime}(m), \xi_{m}$ are given by (8), (9) respectively.

Necessity: Adding (12) over the users $m \in u$ and using assumption (6) with (1), (2) we obtain (10).

Sufficiency: Assume now that for some $\left(w_{m}\right)$ satisfying (4) condition (10) holds true. Then, the power allocation given by (11) satisfies (12) and obviously (6).

Corollary 4.3: If for a given vector of bit-rates $\left(r_{m}\right)$, there exist bandwidths $\left(w_{m}\right)$ satisfying (4) and (10) then $\left(r_{m}\right)$ is feasible.

Proof: Assume first that each base station has at least one user. Then, by Proposition 4.2, $\left(r_{m}\right)$ is feasible at maximal power and thus is feasible (note that (6) implies (3)).

In a case when some base stations, say in $U^{\prime} \subset U$ have no users, then for them the feasibility problem does not exist. Consider the remaining base stations and the given vector of their bit-rates $\left(r_{m}\right)$ for $m \in u \in U \backslash U^{\prime}$. Take some bandwidths $\left(w_{m}\right)$ satisfying (4) and (10); by our assumption they exist. Consider the power allocation given by (11). It satisfies (6) and thus (3). By condition (10) it also satisfies (12), which is equivalent to a modification of the information theory condition (5) that consists in replacing $P_{v}$ by $\tilde{P}_{v}, v \in U$. For $v \in U \backslash U^{\prime} P_{v}=\tilde{P}_{v}$ and for $v \in U^{\prime} P_{v}=\epsilon \tilde{P}_{v} \leq \tilde{P}_{v}$. Thus, this modified information theory condition is more constraining, which means that (12) implies (5). This completes the proof.

We call the condition expressed in Corollary 4.3, the first sufficient feasibility condition (SFC1). 
B. When all BS emit with their maximal powers and the users have equal power spectral density

In this section, in addition to the maximal emitted power assumption (6) (when each BS has at least one user), we will assume that the base stations observe the following condition

$$
P_{u, m}=\frac{w_{m}}{W}(1-\epsilon) \tilde{P}_{u}, \quad m \in u, u \in U
$$

which means that the all users have equal power spectral density. Note that under condition (13) conditions (4) and (3) are equivalent. Consequently we will say that:

Definition 4.4: A given vector of user bit-rates $\left(r_{m}\right)$ is feasible at maximal power and equal users' power spectral density if there exist bandwidths $\left(w_{m}\right)$ satisfying

$$
\sum_{m \in u} w_{m}=W, \quad u \in U
$$

and (5) with $P_{u, m}$ given by (13).

Proposition 4.5: A vector of user bit-rates $\left(r_{m}\right)$ is feasible at maximal power and equal users' power spectral density iff

$$
\sum_{m \in u} \frac{r_{m}}{\log _{2}\left(1+1 / f^{\prime}(m)\right)} \leq W, \quad u \in U
$$

Moreover, in this case the following bandwidth allocation

$$
w_{m}=\frac{r_{m}}{\log _{2}\left(1+1 / f^{\prime}(m)\right)} \frac{W}{\sum_{n \in u} r_{n} / \log _{2}\left(1+1 / f^{\prime}(n)\right)},
$$

for $m \in u, u \in U$ satisfies (14) and (5) with $P_{u, m}$ given by (13).

Proof: Recall from the proof of Proposition 4.2 that under the maximal power assumption the information theory constraint (5) is equivalent to (12). Using (13) we can further rewrite it as

$$
\frac{w_{m}}{W} \geq f^{\prime}(m) \xi_{m}=f^{\prime}(m) \frac{w_{m}}{W}\left(2^{r_{m} / w_{m}}-1\right)
$$

which is equivalent to

$$
r_{m} \leq w_{m} \log _{2}\left(1+1 / f^{\prime}(m)\right)
$$

Necessity: Evaluating $w_{m}$ form (17), adding over users $m \in u$ and using (14) we obtain (15).

Sufficiency: Assume (15). Consider the bandwidth allocation given by (16). Obviously it satisfies (14). From (15) and (16) we deduce (17). This completes the proof.

Corollary 4.6: For a given vector of bit-rates $\left(r_{m}\right)$ if condition (15) is satisfied then this vector is feasible.

Proof: This is obvious under the assumption that each base station has at least one user. The proof for a general case goes along the same lines as this of Corollary 4.3.

In this context we call condition (15) the sufficient feasibility condition (SFC). Note that SFC has the desired multi-Erlang form allowing for its analytical evaluation.

\section{BLOCKING PROBABILITIES FOR STREAMING TRAFFIC}

\section{A. Traffic demand}

Denote the geographic region covered by the (multi-)cellular network by $\mathbb{D}$ that is assumed to be a bounded subset of the plane $\mathbb{R}^{2}$. Consider only streaming calls whose inter-arrival times to $\mathbb{D}$ are independent and identically distributed (i.i.d.) exponential random variables with rate $\lambda$ (mean $1 / \lambda)$. The position of each arrival is picked at random in $\mathbb{D}$ according to some distribution $Q(d x)$. We assume that users don't move during their calls. Each call requires to be served by the network at a given bit-rate during some service time. The durations of the different calls are assumed to be i.i.d. exponentially distributed with mean $1 / \mu$. (This assumption may be relaxed due to the so-called insensitivity property, but this is not in the scope of the present paper.) The quantity $\rho(\mathbb{D})=\lambda / \mu$ is called the traffic demand (expressed in Erlangs) in the whole network.

The set of positions of all users served at a given time is called configuration of users. Let $\mathbb{M}$ be the set of all possible configurations (this can be formalized e.g. on the basis of the theory of point processes). We denote by $\left\{N_{t}\right\}_{t \geq 0}$ the process describing the evolution in time of the user configurations in $\mathbb{D}$ (due to arrivals and departures) in the absence of any admission control. It takes its values in $\mathbb{M}$. We call it free process. By our previous assumptions the free process $\left\{N_{t}\right\}_{t>0}$ is a Markov process that is ergodic and has the stationary Poisson distribution $\Pi$ of user configurations on $\mathbb{D}$ with mean measure $\rho(\mathbb{D}) Q(d x)$. ( $\Pi$ describes the user configurations and not only their total number.) Moreover $\left\{N_{t}\right\}_{t \geq 0}$ is reversible with respect to $\Pi$.

\section{B. Elang's loss model}

We assume that a given admission condition consists of verifying whether a given configuration of users with a new arrival belongs to some set of feasible configurations $\mathbb{M}^{\mathrm{f}}$. We tacitly assume also that no user departure from a feasible configuration can make it unfeasible. Denote the evolution of the free process modified (controlled) by the given admission condition by $\left\{N_{t}^{\mathrm{f}}\right\}_{t>0}$. This process is also Markov. More precisely, it has the same dynamics as the free process except that the transitions (i.e. arrivals) that would lead outside $\mathbb{M}^{f}$ are blocked. Such a modification of the Markov process is called truncation (of the free process) to $\mathbb{M}^{\mathrm{f}}$. The crucial observation, made by the reversibility of the free process, is that the truncated process $\left\{N_{t}^{\mathrm{f}}\right\}_{t \geq 0}$ admits as its invariant distribution the truncation of $\Pi$ to $\mathbb{M}^{\mathrm{f}}$; that is, $\Pi^{\mathrm{f}}(\Gamma)=\Pi\left(\Gamma \cap \mathbb{M}^{\mathrm{f}}\right) / \Pi\left(\mathbb{M}^{\mathrm{f}}\right)$ for $\Gamma \subset \mathbb{M}$.

The blocking probability (in some region of the network) is defined as the proportion of the blocked calls to the total number of calls arriving to this region in the long run of the system. The celebrated Erlang's loss formula allows to express this ergodic average by means of the invariant measure of the free process and in our spatial point process formalism takes the form $b_{m}=$ $\Pi\left(\left\{\nu \in \mathbb{M}^{\mathrm{f}}: \nu+\varepsilon_{m} \notin \mathbb{M}^{\mathrm{f}}\right\}\right) / \Pi\left(\mathbb{M}^{\mathrm{f}}\right)$, where $b_{m}$ is the blocking rate of users arriving at the location $m \in \mathbb{D}$ and $\nu+\varepsilon_{m}$ denotes configuration $\nu$ appended with one user located at $m$ (cf [4]). Integrating $b_{m}$ against the distribution $Q(d m)$ over some given subset of the network $\mathbb{D}$ gives the blocking probability in this set (region) of the network.

For this formula to be of any use in the dimensioning process one needs an efficient way of evaluating the Poisson probabilities in the numerator and the denominator, in particular $\Pi\left(\mathbb{M}^{f}\right)$. Such efficient method exists for some particular form of the admission condition as we explain in what follows.

We say that the admission condition has the multi-Erlang form if the corresponding set of feasible configurations $\mathbb{M}^{f}$ has the following form

$$
\mathbb{M}^{\mathrm{f}}=\bigcap_{u \in U}\left\{\nu \in \mathbb{M}: \sum_{m \in u, m \in \nu} \varphi_{m}^{u}<C_{u}\right\}
$$

where $U$ is the set of all base stations, the sum of the values of some function $\varphi_{m}^{u}$ is taken over of users $m$ in configuration $\nu$ and served by BS $u$. This function may depend on the user $m$ geographical location, its bit-rate and also on the serving base station. $C_{u}$ is some constant, possibly dependent on the base station. In this case, we may easily evaluate the blocking probability by discretization of $\mathbb{D}$ and using the KaufmanRoberts algorithm [13, 20]. Note that the sufficient feasibility condition (15) has the multi-Erlang form (18). 

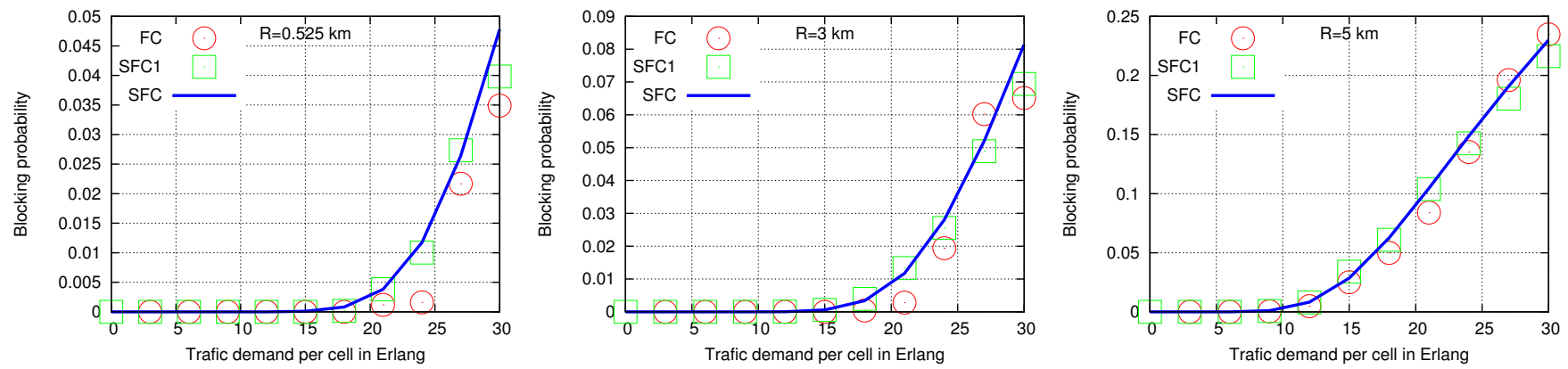

Fig. 1. Blocking probability imposed by various admission conditions for the network with cell radius $R=0.525,3,5 \mathrm{~km}$.

\section{Validation of the sufficient condition}

1) Model specification: In order to obtain numerical values, we consider the most popular hexagonal network model, where the base stations are placed on a regular hexagonal grid. Let $R$ be the radius of the disc whose area is equal to that of the hexagonal cell served by each base station, and call $R$ the cell radius. Bearing in mind the above pattern of base stations, one considers $\mathbb{D}=\bigcup_{u} \mathcal{C}_{u}$, where $\mathcal{C}_{u} \subset \mathbb{R}^{2}$ are hexagonal cells constituting the network. More specifically, we consider $4 \times 4=16$ toroidal hexagonal cell model. Thus $U$ consist of 16 BS. We assume a path loss $L(r)=(K r)^{\eta}$, with $\eta=3.38$, $K=8667$. This means that the path loss between BS $u$ and user $m$ is equal to $L\left(\left|x_{u}, x_{m}\right|\right)$ where $x_{u}, x_{m} \in \mathbb{D}$ denote, respectively, the geometric location of $u$ and $m$ and $|\cdot|$ is the Euclidean distance.

We consider a streaming traffic of the mean call duration $1 / \mu=2 \mathrm{~min}$ with the required bit rate $180 \mathrm{Kbps}$. Users arrive (spatially) uniformly to $\mathbb{D}$ with intensity $\lambda$. We consider different values of $\lambda$ such that the traffic demand per cell $\lambda \pi R^{2} / \mu$ varies from 0 to 30 Erlangs.

The BS maximal total power is $\tilde{P}=52 \mathrm{dBm}$, the common channel power $P^{\prime}$ is the fraction $\epsilon=0.12$ of $\tilde{P}$ and the ambient noise power $W N_{0}=-103 \mathrm{dBm}$.

2) $S F C$ versus $F C$ : Figure 1 shows the blocking probability per cell in function of the traffic demand for three different admission conditions: FC, SFC1 and SFC, for cell radii $R=$ $0.5,3$ and $5 \mathrm{~km}$. The curves for $\mathrm{FC}$ and $\mathrm{SFC} 1$ are obtained by long simulations ${ }^{7}$ (several days on a typical PC) while this of SFC is obtained by the Erlang's loss formula and KaufmanRoberts algorithm, which takes only a few seconds.

The following important observations can be made: the blocking probability induced by SFC is close to this of SFCl that in turn is sufficiently close to this of FC. The maximal loss of capacity between FC and SFC, i.e. of the traffic demand that can be served at the blocking probability close to 0.02 , is about $10 \%$. We conclude that SFC, whose performance can be evaluated analytically, is a good basis for the dimensioning process.

\section{CONCLUSION}

We have proposed a dimensioning method for the downlink of OFDMA cellular network (like LTE, WiMAX). It is much faster than simulation since it is based on some multi-rate Erlang loss model, whose blocking probabilities can be evaluated by means of the Kaufman-Roberts algorithm. It is also accurate enough, since it induces only up to (acceptable by future LTE operators) $10 \%$ loss of capacity with respect to a theoretical reference network control.

${ }^{7}$ For this both problems are transformed into an optimisation one (of the type inf of some function to be negative) that is solved by the sub-gradient method.

\section{REFERENCES}

[1] R. Agarwal, V. Majjigi, Zhu Han, R. Vannithamby, and J. Cioffi. Low complexity resource allocation with opportunistic feedback over downlink OFDMA networks. IEEE JSAC, 26:1462-1472, 2008.

[2] F. Baccelli, B. Błaszczyszyn, and F. Tournois. Downlink capacity and admission/congestion control in CDMA networks. In Proc. of IEEE INFOCOM, San Francisco, 2003.

[3] F. Baccelli, B. Błaszczyszyn, and M. K. Karray. Up and downlink admission/congestion control and maximal load in large homogeneous CDMA networks. MONET, 9(6):605-617, December 2004.

[4] F. Baccelli, B. Błaszczyszyn, and M. K. Karray. Blocking Rates in Large CDMA Networks via Spatial Erlang Formula. In Proc. of IEEE INFOCOM, 2005.

[5] B. Błaszczyszyn and M. K. Karray. Performance Evaluation of Scalable Congestion Control Schemes for Elastic Traffic in Cellular Networks with Power Control. In Proc. of IEEE INFOCOM, 2007.

[6] B. Błaszczyszyn and M. K. Karray. An efficient analytical method for dimensioning of CDMA cellular networks serving streaming calls. In Proc. of ValueTools, 2008.

[7] F. Delcoigne, A. Proutière, and G. Régnié. Modelling integration of streaming and data traffic. In Proc. of ITC-SS IP Traffic, 2002.

[8] S-E. Elayoubi, O. Ben Haddada, and B. Fourestie. Performance evaluation of frequency planning schemes in OFDMA-based networks. IEEE Trans. Wireless Commun., pages 1623-1633, 2008.

[9] M. Ergen, S. Coleri, and P. Varaiya. QoS aware adaptive resource allocation techniques for fair scheduling in OFDMA based broadband wireless access systems. IEEE Trans. Broadcasting, 49:362-370, 2003.

[10] S. Gault, W. Hachem, and Ph. Ciblat. Performance Analysis of an OFDMA Transmission System in a Multi-Cell Environment. IEEE Trans. Commun., 2007.

[11] J. Huang, V. Subramanian, R. Agrawal, and R. Berry. Downlink scheduling and resource allocation for OFDM systems. In Proc. of CISS, 2006.

[12] M. K. Karray. Analytic evaluation of wireless cellular networks performance by a spatial Markov process accounting for their geometry, dynamics and control schemes. PhD thesis, ENS, 2007.

[13] J. S. Kaufman. Blocking in a shared resource environment. IEEE Trans. Commun., 29(10):1474-1481, 1981.

[14] Keunyoung Kim, Youngnam Han, and S-L. Kim. Joint subcarrier and power allocation in uplink OFDMA systems. IEEE Communications Letters, 9:526-528, 2005.

[15] D. Kivanc, Guoqing Li, and Hui Liu. Computationally efficient bandwidth allocation and power control for OFDMA. IEEE Tran. Wireless Commun., 2:1150-1158, 2003.

[16] Jun Li, Amitava Bose, and Yiqiang Q. Zhao. Rayleigh flat fading channels' capacity. In Proc. of IEEE CNSR, pages 214-217, Washington, 2005.

[17] Y. Li and G. L. Stuber. Orthogonal Frequency Division Multiplexing for Wireless Communications (Signals and Communication Technology). Springer-Verlag, New York, 2006.

[18] M. Pischella and J-C. Belfiore. Achieving a frequency reuse factor of 1 in OFDMA cellular networks with cooperative communications. In IEEE VTC Spring, pages 653-657, 2008.

[19] W. Rhee and J. M. Cioffi. Increase in capacity of multiuser OFDM system using dynamic subchannel allocation. In Proc. of VTC Spring, pages 10851089, 2000

[20] J. W. Roberts. A service system with heterogeneous user requirements. In G. Pujolle, editor, Performance of Data Communications Systems and their Applications, Amsterdam, 1981. North Holland Pub. Co.

[21] Zukang Shen, J.G. Andrews, and B.L. Evans. Adaptive resource allocation in multiuser OFDM systems with proportional rate constraints. IEEE Trans. on Wireless Commun., 4:2726-2737, 2005.

[22] I.C. Wong, Zukang Shen, B.L. Evans, and J.G. Andrews. A low complexity algorithm for proportional resource allocation in OFDMA systems. In Proc. of IEEE Signal Processing Systems, 2004.

[23] G. Wunder and C. Zhou. Queueing Analysis for the OFDMA Downlink: Throughput Regions, Delay and Exponential Backlog Bounds. IEEE Trans. Wireless Commun., 2009. 